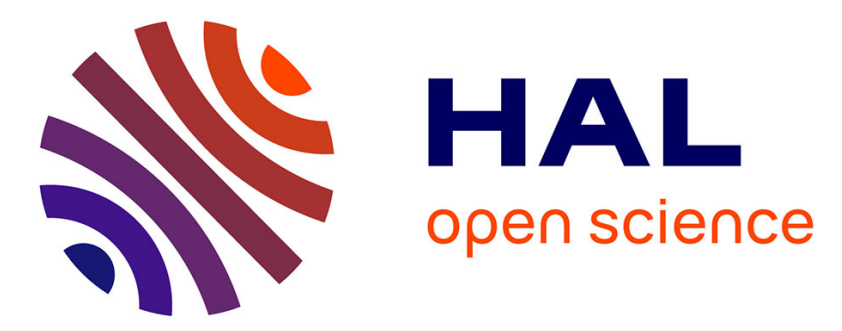

\title{
Wiki for knowledge sharing, a user-centred evaluation approach: A case study at STMicroelectronics
}

Manel Brichni, Nadine Mandran, Lilia Gzara, Sophie Dupuy-Chessa, David Rozier

\section{- To cite this version:}

Manel Brichni, Nadine Mandran, Lilia Gzara, Sophie Dupuy-Chessa, David Rozier. Wiki for knowledge sharing, a user-centred evaluation approach: A case study at STMicroelectronics. Journal of Knowledge Management, 2014, 18 (6), pp.1053. hal-01457730

\section{HAL Id: hal-01457730 \\ https://hal.science/hal-01457730}

Submitted on 10 Feb 2017

HAL is a multi-disciplinary open access archive for the deposit and dissemination of scientific research documents, whether they are published or not. The documents may come from teaching and research institutions in France or abroad, or from public or private research centers.
L'archive ouverte pluridisciplinaire HAL, est destinée au dépôt et à la diffusion de documents scientifiques de niveau recherche, publiés ou non, émanant des établissements d'enseignement et de recherche français ou étrangers, des laboratoires publics ou privés. 


\title{
WIKI FOR KNOWLEDGE SHARING, A USER-CENTRED EVALUATION APPROACH: A CASE STUDY AT STMICROELECTRONICS
}

\author{
Manel Brichni ${ }^{1,2,3}$, Nadine Mandran ${ }^{1}$, Lilia Gzara ${ }^{2}$, Sophie Dupuy-Chessa ${ }^{1}$, David Rozier ${ }^{3}$
}

1- LIG, Univ. Grenoble Alpes, CNRS

2- GScop, INPG

3- STMicroelectronics

Structured Abstract: The purpose of this case study paper is to evaluate how Wiki tools are used for knowledge sharing within industrial organizations. It explores strengths and weakness of wiki tools regarding knowledge sharing, through a case study, in a French company. A Wiki has been deployed since several years within STMicroelectronics Company in order to improve Business Intelligence teamwork. The aim of this research is to evaluate this Wiki performance regarding knowledge sharing objectives. The proposed evaluation methodology is based on a usercentered approach.

Keywords: Knowledge sharing, Wiki, User-centered evaluation approach

Article Classification: Case study 


\section{Introduction}

To preserve employees' own knowledge, some companies adopt various tools for knowledge sharing (Ayres \& GarciaPerez, 2010; Avram, 2006), such as Wiki, Social Bookmarking, collaborative filtering and social networks. Wiki is considered as one of the most important social software. That is the center of our interest in this paper.

A Wiki could be defined as a set of linked pages accessible by users for editing contributions; a simple tool, easy to use and access. It does not require any technical competence. A simple user guide suffices (Lamb, 2004). It allows users to post their knowledge on a common area and to present the collaborative advantage to discuss on it the existing knowledge (Parker Kevin \& Chao, 2007; Gee-Woo, et al., 2005). According to (Lamb, 2004), Wiki stimulates writing, promotes effective communication and low cost collaboration. It also promotes close reading, revision, and tracking of drafts. In (Raitman, et al., 2005), authors described the Wiki as a democratic tool where users can contribute without consequence in order to enrich its knowledge value. Thanks to the contribution of each user, the result is of high value making it more relevant and ensures its evolution (Kaplan-Leiserson, 2004; Teck Heng \& Marimuthu, 2011). These features make Wikis interesting tools.

The objective of this paper is to evaluate performance of such a tool regarding knowledge sharing objectives by analyzing a concrete case study. This case study refers to the IT department at STMicroelectronics company ['] which adopted a Wiki called "Stiki" to enhance capitalization and sharing of Business Intelligence knowledge. This analysis is mainly based on empirical experiments evaluating the way Stiki is used, its weaknesses and strengths. For this aim, a user-centered approach is proposed. This approach integrates two families of methods: qualitative and quantitative, that we will describe later.

In the first part of the paper, the problem space will be defined based on a literature review on Wikis use (section 2) and the case study at STMicroelectronics will be presented (section 3). The second part will focus on the Wiki evaluation by outlining the evaluation process (section 4) and presenting the experimental results (section 5). A conclusion in section 6 ends this paper.

\section{Problem space}

In order to identify our problem space, a set of criteria that should be met by a Wiki is identified. To this end, a literature review of application cases studying the use of Wiki for knowledge sharing in different domains was conducted: In manufacturing industry (Lykourentzou, et al., 2010) with a Wiki called CorpWiki to select the most appropriate person to improve the quality of an article and consequently ensures its relevance; in education, (Krebs, et al., 2010; Ramanau \& Fawei, 2009) to encourage a group of students specialized in Japanese language creating learning pages; in research (Ayres \& Garcia-Perez, 2010) to enhance collaborative work among a group of researchers and in commerce, by the case of eBay (Tay Pei Lyn, 2009; MacManus, 2006) to share commercial knowledge.

From the study of these application cases, two aspects may be considered to evaluate a Wiki: social and technical. Social aspects concern either collaborative or individual dimensions. Technical aspects concern either the Wiki container (tool) or content dimensions. As depicted in Figure 1, a set of criteria according to these dimensions is identified with dotted axis. 


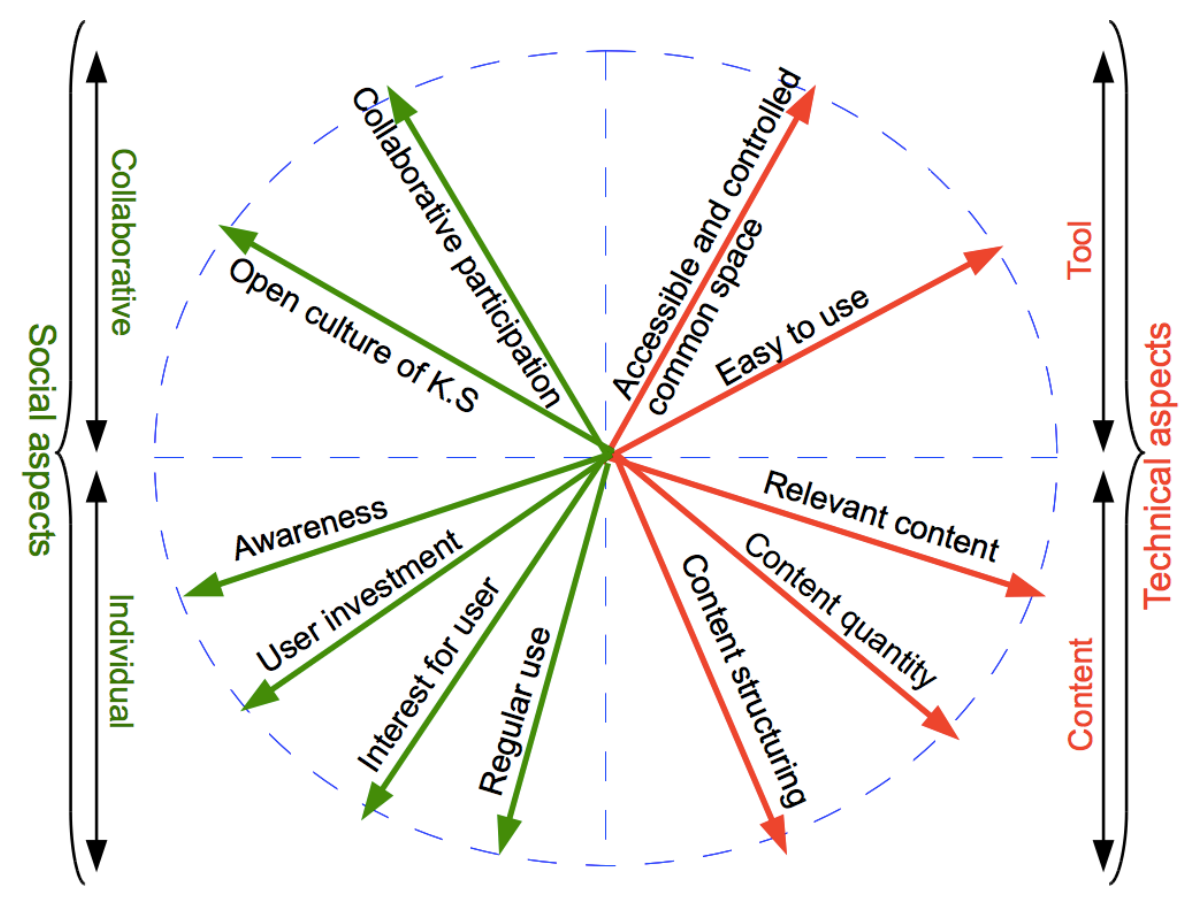

Figure 1 Wiki evaluation criteria

\section{Technical aspects}

Wikis can be evaluated according to two dimensions: the characteristics and functionalities of the tool allowing its use and its content.

Tool dimension: as shown in Figure 1, providing an accessible and controlled common space is considered as one criterion characterizing social software and particularly Wikis. As mentioned in (Ramanau \& Fawei, 2009), not having an easy direct link to the Wiki limits its use and was one of the reasons explaining the decline in its use. Usually, users of Wikis are expecting to deal with a tool, which facilitates their work in a timely and reliable manner. Then, they can easily retrieve the knowledge they search for, and easily transmit to others their own one. In addition to its accessibility, such a tool needs to be controlled since it supports a shared space. Users must have intermediate spaces between public and private use to effectively manage their contributions. Ease of use is one of the criteria that the use of Wiki must also meet (Figure 1). It was a criterion on what the case study in commerce (MacManus, 2006) was based.

Content dimension: in (MacManus, 2006), regarding an e-commerce Wiki, organizing information insured its content relevance. This criterion is also highlighted in (Lykourentzou, et al., 2010) regarding the ability of the proposed Wiki to select the most appropriate person who will improve the quality of the article and then ensures its content relevance. That explains why in Figure 1 we integrate content relevance as a criterion the Wiki must meet. Dealing with content relevance implies introducing content quantity. This criterion consists of providing enough knowledge so that Wiki' users can perform their tasks by reusing existing one and enriching their own one. Such a criterion is highly dependent on users' behavior and particularly their contributions. Besides, we were inspired by a study about capitalizing software design knowledge with design patterns (Conte, et al., 2001). The design pattern community provides a formalism for patterns representation called P-Sigma (Conte, et al., 2001). Such formalism aims at making a uniform expression of product patterns and process patterns allowing a better organization of their libraries. For the same reasons, we consider that content structuring is an important criterion. As the common formalism of patterns makes explicit their selection interface, the page content structure guides users to correctly contribute and to efficiently search for information. That explains why we integrate in Figure 1, content relevance, content quantity and structured pages content as content criteria.

\section{Social aspects}

Wikis can be evaluated according to: an individual dimension (user behavior towards its use) and the collaborative dimension (behavior of all users interacting through it). 
Individual dimension: in (Ayres \& Garcia-Perez, 2010), authors argued that because there was very little interaction among researchers on a regular basis, knowledge sharing via Wiki was not taking place regularly. This highlights issues relating to the lack and non-regularity of use. Furthermore, irregularity of use is usually due to lack of awareness regarding knowledge sharing benefits and consequently interest for Wiki use. That was the case of research' Wiki described in (Ayres \& Garcia-Perez, 2010): the impression that Wiki does not have much to offer for those who are in the organization since long time does not motivate them to invest time in using it. However, in the case of education' Wiki described in (Ramanau \& Fawei, 2009), although using Wiki presented an important interest for each one, students did not investigate as expected. That's why awareness and interest are reasons why users invest less time in sharing knowledge than in other works. These observations explain the integration of these criteria in Figure 1.

Collaborative dimension: knowledge sharing is only valid if Wiki is collectively used. The case of CorpWiki in the manufacturing industry case (Lykourentzou, et al., 2010) includes a 'fairness-based" policy, where the selection is made based on a fair workload distribution, ensuring then collaborative participation. Finally, knowledge sharing, particularly in a large organization, requires an open culture. That means naturally including collaboration at work. In the case of commerce Wiki (MacManus, 2006), employees have to adapt themselves to the online trading concept and learn the culture and ways of working of eBay. That was one of the success keys of eBay experience in using Wiki for knowledge sharing. In Figure 1, these criteria correspond to the collaborative part of our problem space.

Therefore, based on the literature review and criteria described above, Wiki deployed within STMicroelectronics Company is evaluated. In the following, STMicroelectronics Wiki is first described (section 3 ) and then proposed evaluation process is developed (sections $4 \& 5$ ).

\section{STMicroelectronics Wiki}

STMicroelectronics is a global leader in semiconductors. It is the largest European company in its field. It has existed since 1987, after a merge between the Italian company SGS Microelettronica and the French company THOMSON Semiconductor.

The IT (Information Technology) department at STMicroelectronics has opted for a Wiki for knowledge sharing, called Stiki. It was created at Crolles 300 site in France by the Manufacturing Solutions Group, having almost 75 employees and around 60 subcontractors. This group contains different teams such as internal architecture, business intelligence, production systems and automation. The main missions of this group are to support, maintain and adapt over time Crolles 300 information systems to the level required by a world class $300 \mathrm{~mm}$ F Fabrication and R\&D plant (Research and Development), as well as enrich Crolles300 information systems with the integration of new software modules needed by the latest semiconductor technologies. Besides, Stiki has currently 240 registered users belonging not only to the Manufacturing Solutions Group but also from other teams (internal architecture, business intelligence, production systems and automation, etc.), with an administrator who is frequently changed, where $48 \%$ of them are searching in Stiki and $71 \%$ made revisions to existing content at least once.

To launch the official department wide use of Stiki, first, a working group was established only with the launcher of Stiki and the assigned administrator, to test it and create first pages. Next, another larger working group was made of one person of each team (managers since they are responsible for communication within their teams) to discuss the desired content to add in Stiki according to their needs. Therefore, some templates were proposed to guide the users' contributions. Finally, a public meeting was held, during which a presentation of the tool and its use took place. Moreover, during its use, teams' evolution and internal reorganizations were also a way to expand the scope of application of Stiki. For example, an employee from the team "IT Manufacturing Execution Systems" was transferred to the team "Capacity \& Equipment Productivity", where Stiki was not known. She proposed then its use.

\section{Stiki: Design and structure}

Stiki has been designed and implemented in 2009 to cover the support procedures, technical, business and functional documentation. Deploying Stiki particularly aims at overcoming problems due to the lack of knowledge sharing, existence of different repositories, hard searching for relevant information and writing documentation. It is implemented using MediaWiki package.

Stiki' content targets two types of use: a public use (main part) for all STMicroelectronics employees and a private use (support part) for IT support team only. Hence, an IT support team member can describe an application either in the main part from a functional point of view (by explaining its functionalities and describing application' architecture and technical design) or in the support part from a technical point of view (by providing various support procedures where he explains how to deal with problems related to applications development or maintenance).

Furthermore, the subject of structuring content pages of Stiki was discussed. We define the structure of page content by the organization of its information with a common formalism. For example, a page describing an application development guide should provide information about prerequisites, development set-up, tests and application delivery. 
Content structuring can not only reduce access time to information, but also can help users to correctly transmit information in a formal way. To this end, in Stiki, the content of each page should correspond or be adapted to an appropriate template. Each template describes a part of the system from a design, architecture, and development or support point of view and guarantees the overall consistency of the page content. For example, the template of the technical design demonstrates the modeling of the system and the development guide template describes the prerequisites, the development set up, the acceptance tests, etc.

\section{Proposed evaluation process: a user-centered approach}

Studies, about the evaluation of Wiki' use within an organization, are limited. They are either related to the adopted assessment methods (Montero-Fleta \& Perez-Sabater, 2011), or the obtained results (Ramanau \& Fawei, 2009). The case of CorpWiki (Lykourentzou, et al., 2010), for example, addresses the process of setting up a Wiki to support knowledge sharing. In our case, the Wiki already exists. To conduct this experiment, four researchers conceived the followed strategy and two industrial engineers provided the working environment. Based on a user-centered approach, the evaluation methodology proposed in this research aims at understanding how Stiki is used, by collecting users' opinions about its advantages, limits and areas of progress. It takes into account current practices and considers Wiki users as the main source of information. Moreover, it incorporates both qualitative and quantitative methods, closely linked:

- Qualitative Methods: address more precisely the meaning that each individual brings to events. (Sandelowski, 1996) suggests that qualitative data can provide insight on how the participants actually use and respond to intervention, which may vary from one person to another and may be different from the perception of its use and the effectiveness of investigators. Face-to-face interviews, focus groups, forums, blogs and text analysis are examples of qualitative methods. They aim at deeply understanding users and their environment and at identifying a maximum of uses, behaviors and opinions with a maximum of variability (Duyck, 2003).

- Quantitative Methods: aim to quantify and to measure the performance of tools. Their strength is that they are based on factual and reliable results that are usually generalized to a large population (Fonteyn \& Bauer-Wu, 2005), mainly statistical analysis methods, performance tests and a questionnaire SUS (System Usability Scale) (Brooke, 1996). They allow a quantitative analysis to understand the reasons behind found facts and collect suggestions for improvement for the evaluated tool.

\section{Process stages description}

Then, the proposed assessment process follows these stages (cf. Figure 2):

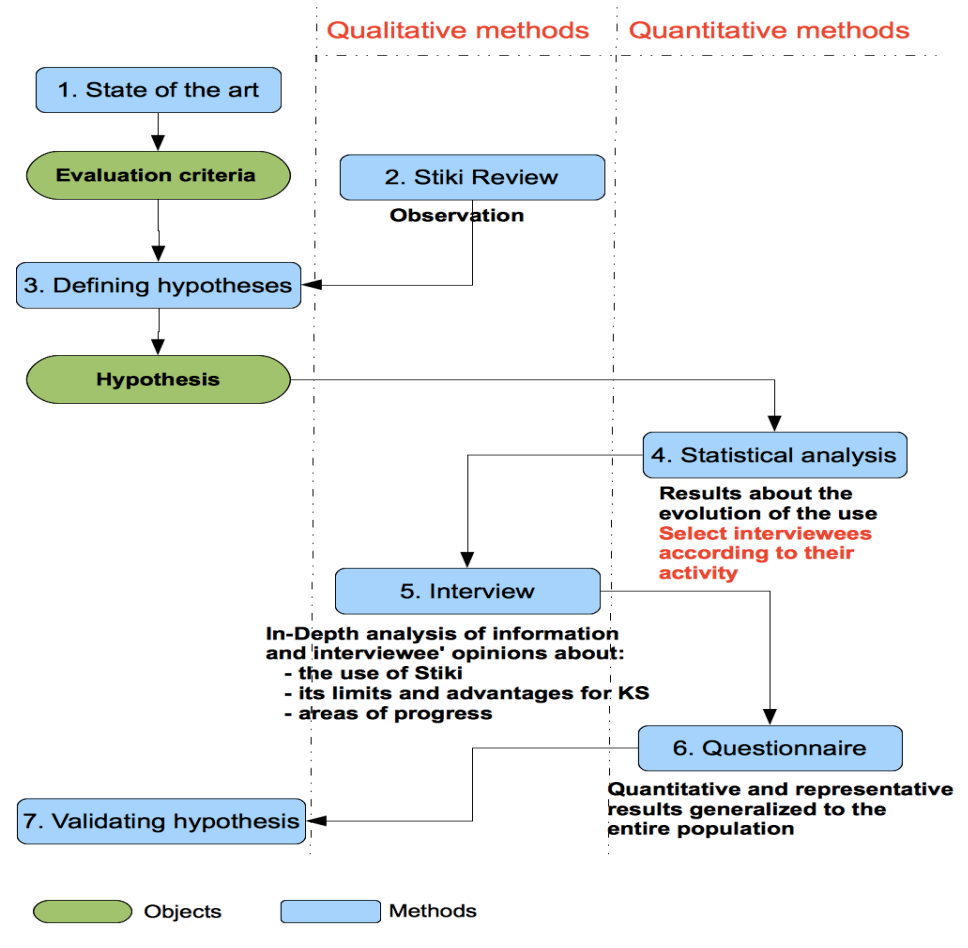

Figure 2 Stages of our proposed assessment process 
1. Evaluation criteria identification: This helps specifying the axis on which we focus our evaluation, as well as selecting appropriate assessment methods. This step is considered as a preparatory step before starting with evaluation methods. Identified criteria have been described in section 2.

2. Stiki Review: this weekly meeting established at the beginning of the evaluation process aims at observing and discussing how Stiki users proceed in their use during each week. The goal is to test the responsiveness of Stiki users regarding our observations. The principle is neither to give nor to get orders. Such meeting allows to observe users' behavior and then to build a preliminary vision on Stiki' use. Evaluation criteria (identified in step 1) are considered during Stiki review in order to make a first evaluation of these criteria.

3. Defining hypotheses and questions: this step is a key element of the proposed approach. The aim of this step is to assess the use of Stiki according to each evaluation criterion. To do that, when we have an idea about the criterion (mainly from the Stiki Review), a hypothesis to be verified is built; otherwise a question to be answered is defined in order to evaluate the concerned criterion. In that way, the criteria are transformed to hypothesis and questions. Each hypothesis or question treats one criterion and contributes to its evaluation, where each one could be validated thanks to the obtained results of one or more evaluation methods. The list of proposed hypotheses and questions is given in the following, classified according to the group of dimensions they refer to $(\mathrm{H}$ : hypotheses and $\mathrm{Q}$ : questions):

a. Tool dimension:

- H1: Stiki is an accessible and a controlled common space

- H2: Stiki is easy to use

b. Content dimension:

- Q3: Is Stiki content relevant?

- Q4: Is there sufficient content of information in Stiki?

- Q5: Structuring pages has an impact on the visibility of its content and facilitates the search and writing of information in the appropriate place. Is it mastered at STMicroelectronics?

c. Individual dimension:

- H6: Stiki users are aware regarding the importance of knowledge sharing as well as the role of Stiki to improve it

- H7: Despite the awareness of Stiki' users regarding the importance of knowledge sharing, they do not invest enough time in its use

- Q8: Is the use of Stiki interesting for users to share their knowledge?

- Q9: Does each user share knowledge regularly?

d. Collaborative dimension:

- Q10: Did collective participation and collaborative management of pages exist? Are individual pieces of knowledge available to others? Is modification of one's own writing by others well received?

- Q11: Is STMicroelectronics' culture favorable to improve knowledge sharing?

4. Statistical analysis: the access to the Stiki database and extraction of statistic information related to the defined hypothesis and questions allow understanding how Stiki' use has evolved. It also allows performing descriptive statistical analysis on some aspects such as:

a. The evolution of visits to Stiki per week or per month This allows determining how the use of Stiki has evolved over time. Depending on the type of use, other measures are identified.

b. Contribution: number of contributions and contributors per page

This serves measuring the activity on each page and then identifying those with single contributor, for example, to understand what type of defaults these pages have and need reviewing.

c. Consultation: number of readers per page

These measures could be used to verify the utility of Stiki among employees benefiting from its content.

d. Modification: the activity of users in revision

This measure allows us classifying users according to their activity and then, varying the selection of users for the individual interviews.

5. Semi-structured individual interviews: We aimed at gaining insights from users' point of view about the use of Stiki for sharing knowledge. 
To this end, a list of participants for interviews is defined based on their activity on Stiki (thanks to the statistical measure classifying Stiki users from the least active to the most active one). The idea is to vary all of them (240 users) according to their activity on Stiki (consultation and contribution), equitably distributed on four categories "not active, relatively active, active and very active". Their membership to different teams is also considered. The idea here is to vary users according to their work and functions (internal architecture, business intelligence, production systems and automation, etc.). Twenty users were selected from the site Crolles 300. The interviewees belong to different categories of age, from 20 to over than 40 years old, and are picked from five different teams (Four employees of each team where each one belongs to a category of contributors - from the less active to the most active contributor). Such a selection allows having a representative sample of all the users.

The interviews lasted between 30 minutes and an hour depending on the interest of users in the experimentation and their activities on Stiki. An individual analysis grid summarizing interviewer' answers and ideas are built after each interview. During the interview, explored topics and questions should be related to defined hypothesis and questions (step 3) without constraining interviewees to a particular format.

6. The online questionnaire invitation: Results obtained through the semi-structured individual interviews cannot be quantifiable or generalizable to the whole population. That is why a questionnaire based on interviews results was proposed. It contains questions of different natures (Yes/No questions, multiple choices questions, open questions and leading questions). The questionnaire was mainly designed for Stiki users, to quantify interviews' results and opinions on its use. Collected answers are then used to confirm hypothesis or answer questions defined in step 3. Among 200 users invited to participate (from the same site Crolles 300 and all teams and functions in the IT section who use or do not use it), 68 answered the questionnaire where twothirds are from the IT section and the others are either co-contactors or do not belong to the IT section.

7. Validating hypothesis and questions: The aim of our process evaluation was to verify finally hypothesis and questions defined previously. Each hypothesis/question (defined in step 3) was validated by one or more evaluation methods. Through the proposed evaluation process, the aim is to employ a user-centred analysis approach. The principle is to study users' ideas, words they use (Verbatim) and associated meaning. The whole process and methods (qualitative and quantitative) allowed validating hypothesis and answering questions.

Before presenting experimental results in the following section, Table 1 shows how each evaluation criterion can be evaluated by one or more methods of our process.

\begin{tabular}{|l|l|c|c|c|c|}
\hline \multirow{2}{*}{ Dimensions } & \multicolumn{1}{|c|}{ Assessment methods } & $\begin{array}{c}\text { Stiki } \\
\text { Review }\end{array}$ & Statistics & Interview & Questionnaire \\
\hline \multirow{2}{*}{ Cool } & Accessible and controlled common space: H1 & & & $\mathrm{X}$ & $\mathrm{X}$ \\
\hline & Ease of use: H2 & & & $\mathrm{X}$ & $\mathrm{X}$ \\
\hline \multirow{4}{*}{ Content } & Relevant content: Q3 & & & $\mathrm{X}$ & $\mathrm{X}$ \\
\cline { 2 - 6 } & Content quantity: Q4 & & $\mathrm{X}$ & & \\
\cline { 2 - 6 } & Content structuring: Q5 & $\mathrm{X}$ & & $\mathrm{X}$ & $\mathrm{X}$ \\
\hline \multirow{4}{*}{ Individual } & Awareness of user: H6 & & & $\mathrm{X}$ & $\mathrm{X}$ \\
\cline { 2 - 6 } & Investment of user: H7 & $\mathrm{X}$ & $\mathrm{X}$ & $\mathrm{X}$ & \\
\cline { 2 - 6 } & Interest for user: Q8 & & & $\mathrm{X}$ & $\mathrm{X}$ \\
\cline { 2 - 6 } & Regular use: Q9 & & $\mathrm{X}$ & $\mathrm{X}$ & $\mathrm{X}$ \\
\hline \multirow{2}{*}{ collaborative } & Collaborative participation: Q10 & $\mathrm{X}$ & & $\mathrm{X}$ & \\
\cline { 2 - 6 } & Open culture of knowledge sharing: Q11 & & & $\mathrm{X}$ & $\mathrm{X}$ \\
\hline
\end{tabular}

Table 1 Evaluation criteria' assessment methods

\section{Experimental results}

In this section, we first discuss the different findings about STIKI use from the evaluation methods. Then, we identify areas of progress. 


\section{Experimental results analysis}

Experimental results are classified according to the dimensions of criteria (Tool, content, individual and collaborative) and then according to hypotheses and questions treating each one. A synthesis of obtained results is presented in Figure 4 where each axis (criterion) is graduated according to the obtained result (Low, Medium and High).

\section{Tool criteria}

\section{H1: Stiki is an accessible and controlled common space}

By studying the use of Stiki, different types of use are reported:

- Contribution: action of adding new information. It includes creation of pages, revision of existing information or deletion. The user doing this action is called contributor.

- Consultation: action of reading existing information. The user doing this action is called reader.

Stiki is a fast and efficient tool which is accessible by everyone. All users highlight its accessibility. An interviewee mentioned the draft option as an efficient way to control Stiki interface and provide an intermediary page between private and public spaces. That explains why in Figure 4, Stiki is considered verifying totally the "Accessibility to a controlled common space" criterion.

\section{H2: Stiki is easy to use}

According to the questionnaire, $80 \%$ of users (who participated to the questionnaire) highlight the ease of use and efficiency of Stiki especially in consultation. An interviewee said, "I can find the information quickly and simply using a keyword for example", another one said "Stiki is an efficient tool; it allows me to share my knowledge quite clearly and effectively".

When asked about its ease of use, $85 \%$ say that they feel comfortable using it. One interviewee said, "Stiki is a modern tool, it has a friendly interface, ensures quick search and is very convenient in case of absence".

However, one interviewee has a preference for another tool which has more advanced functionalities. He said "I use another tool for sharing my knowledge with my team; I can search any information by keyword even by using images and that functionality does not exist in Stiki ". In addition, when asking if they feel comfortable in adding information in Stiki, about $50 \%$ of users find that adding information to Stiki is still difficult. When asking them if they need help when using Stiki, 35\% of them think so. That is why in Figure 4, "Easy to use" criterion corresponds to "Medium" level particularly due to these difficulties in contribution.

\section{Content criteria}

\section{Q3: Is Stiki content relevant?}

When asking users to assign a level of consistency, relevance and utility for knowledge stored in Stiki, more than $75 \%$ of them confirm having confidence in stored knowledge and believe that it is relevant, useful, updated and slightly obsolete. The relevance of information is very important to ensure the usefulness of Stiki. An interviewee said "We need to monitor knowledge update to ensure that it will not become obsolete" especially in semiconductors' manufacturing field, where products change regularly and data can quickly become obsolete. Despite this, some interviewees point out some inconsistencies in Stiki knowledge and claim for regular updating and monitoring. Ensuring a relevant content is one of the most important points highlighted by Stiki users during the interviews. Using it regularly to update existing knowledge or provide new one helps avoiding its obsolescence, reassures readers and motivates them to use it, not only as reader but also as contributors.

According to the database of Stiki, number of visits to all Stiki pages is about 160,000 . However, the number of visits depends on the page. Indeed, some pages are more popular than others and it may be an indicator of the value of the page content. For instance, Support_TPI page belongs to the support part of the page structuring. It contains all links to support procedures. That is why each time a user adds a procedure; he has to indicate it in the common page Support_TPI. This type of page is usually characterized by a big number of consultation and contributions, which is explained by its important interest. In order to keep the relevance of such a page, collaborative participation is strongly required.

According to the questionnaire, more than $40 \%$ of users access to Stiki once a month or less, seeking for information. Some people would consult only to raise a doubt. Others have preferences for other search tools and criticize its limited guidance for IT.

Besides, the average of consultations per page is 76 , whereas the standard deviation is about 473 . This indicates that a big number of existent pages are not consulted and could potentially encounter problems such as content obsolescence, as some interviewees proclaim. These findings explain why in Figure 4, Stiki corresponds to the "High" 
level for "Relevant content" criterion and not the "Advanced" one due to the existence of some obsolete content that should be treated.

\section{Q4: Is there sufficient content of knowledge in Stiki?}

Today, Stiki contains 2370 pages. All interviewees considered that the number of pages is sufficient compared to the number of realized projects. However, many of them found that the content still targets IT applications without discussing enough functional and operational area. In this context, an interviewee said "In Stiki, we find mainly information about IT applications and techniques but rarely functional and operational information to understand why an application exists, what its purpose is and who is the involved staff". That is why; they found that even though there is a sufficient volume of information in Stiki, it does not really include all domains as it should do. Moreover, when asked if Stiki may be useful for users outside the IT, $85 \%$ of the questionnaire participants agreed and many of them proposed expanding its perimeter of application of Stiki as an area of progress that should be studied. In addition, when asked about their professions in the questionnaire, $70 \%$ of participants are IT staff that shows the limitation of its perimeter application.

That explains why in Figure 4, Stiki corresponds to "High" level for "Relevant quantity" criterion.

\section{Q5: Structuring pages has an impact on the visibility of its content and facilitates the search and writing of information in the appropriate place. Is the structuring of pages mastered?}

The majority of users consider that structuring pages in the form of templates is a good idea. According to the questionnaire, when we asked users about the degree of their agreement with the utility of structuring pages, on average, $80 \%$ of users find that the information structure helps, guides search, unifies content and improves the understanding of the information. Given that the proposed guide for structuring helps users, $65 \%$ of them think that it is applicable, easy to follow and adaptable to particular cases. Such a structure is a kind of guide for users facilitating use of Stiki for searching and contributing information. An interviewee said for this "providing a guide for structuring pages could only be beneficial".

To launch the official department wide use of Stiki, a meeting was held, during which a presentation of the tool and its use took place. This meeting was the only one since its implementation. Several users of Stiki have not assisted for many reasons. One of the guides that the administrator has joined to Stiki is the structure in the form of templates. This structure was also introduced at the meeting launching the activity of Stiki but nevertheless, when asked if they know it, $50 \%$ of users do not. Today, it is one of the reasons preventing some people to use Stiki or causing difficulties during its use. Yet, the majority is confronted to use Stiki regularly. Some make the effort to learn and others use it in their own way. This problem has to be treated by ensuring a regular evaluation of existing problems, a solution proposed by an interviewee, or a regular training, a solution proposed by another. The fact that structuring page is not yet mastered explains why in Figure 4, Stiki corresponds to "Medium" level for "Content structuring" criterion.

\section{Individual criteria}

\section{H6: Stiki users are aware regarding the importance of knowledge sharing as well as the role of Stiki to improve} it

According to the questionnaire, all users have recognized the importance of Stiki for sharing knowledge and despite the existence of other sharing tools, $60 \%$ of them confirmed that Stiki is now the reference tool. In this regard, one interviewee said, "the time spent to produce knowledge on Stiki cannot be considered as a wasted time", another added "Obtaining knowledge about an application of another team via Stiki saves me time without wasting others' time". That explains why in Figure 4, "users' Awareness" criterion corresponds to the "high" level.

\section{H7: Despite the awareness of Stiki' users regarding the importance of knowledge sharing, they do not invest enough time in its use}

Several interviewees consider that documenting their work is optional, secondary and that it takes time. One interviewee said that it is not part of the missions endorsed to him by his manager. Another one has even reported that he often uses Stiki as a knowledge base but he does not document his own work. Three other interviewees think so. Unfortunately, when asked in the questionnaire about the frequency of contribution in Stiki, 30\% of users have never contributed and $55 \%$ contribute approximately one a month for several reasons. Two users consider that there is no necessity, another one considers that he does not have sufficient knowledge to contribute or update existing one, may be due to fear of judgment or fear to sell their expertise and losing their job. Someone else said that there is no knowledge to add. Finally, some of them do not have enough time for that. These different observations led to consider that "Investment of users" criterion corresponds to the "Low" level in Figure 4.

\section{Q8: Using Stiki present an interest for users}


According to the interviews, $90 \%$ of the interviewees consider it as a useful tool in their work and confirmed that Stiki saves time. For four interviewees, searching or contributing information on Stiki has become a reflex and a habit. An interviewee said that "Documenting on Stiki becomes a reflex", another one said "it becomes a reflex to seek information first on Stiki, and then to contact directly the appropriate person". Knowing the interest of such a tool encourages employees to use it and then ensure the increase in its use. However, $10 \%$ the interviewees does not find interest because they use other tools to share or think that its content is intended for a specific population. That is why, as shown in Figure 4, despite the awareness of users of its interest for knowledge sharing within the organization, Stiki "Interest for user" criterion corresponds to "Medium" level, identifying here an improvement to seek.

\section{Q9: Does user share his knowledge regularly?}

Since first implementation of Stiki in 2009, a significant variation in number of contributions is observed. The total number of contributions is 7193 in 2370 pages. Stiki has 230 users including administrator. However, only 147 are considered as real contributors (who participated at least one for contributing) from different services of MSG (Manufacturing Solutions Group) described above. The number of contributions varies according the period and users' need to contribute; generally related to projects' period.

First, just after the period of its launch, Stiki encountered a recession period during which its use had recessed to 0 during three months. Then, throughout 2009, Stiki encountered a slight variation on the number of contribution varying between 20 and 100 actions per month. Then, during 2010, a gradual and significant increase appeared to reach a peak of 400 actions on Stiki in July 2010 and stabilized in the first half of the year 2011. This peak is explained by a strong documentation for some projects developed in this period. Later, during 2012, the number of contributions is stabilized (around 100 actions per month). However, during the year 2013, it had increased. This can be explained by the launch of our work on Stiki assessing, especially during the "Stiki Review" period (cf. evaluation process described in Section 4).

According to the questionnaire, more than $55 \%$ of users contribute once a month. Moreover, the majority of interviewees contributes to Stiki for most projects and particularly shares their production procedures. Only a few of them use it as an own personal memory where they find and store their work. Even though users regularly use Stiki to search for information, it is less used for contribution. That explains why in Figure 4, "Regular use" criterion corresponds to the "Medium" level.

\section{Collaborative criteria}

\section{Q10: Did collective participation and collaborative management of pages exist? Are individual knowledge available to others? Is modification of one's own writing by others well received?}

The level of contribution may be shown not only by the number of contributions, but also by the number of users participating in such contributions. Despite the large number of contributions during certain periods, one can see that very often it comes from the same user. Some examples are given in Figure 3. For example, the page M.E.S has been amended more than 100 times by 12 different users, whereas the page "Dispatching rules" has been amended more than 160 times by only 7 different users. This can be considered as an indicator of page quality. Besides, noting that, on average, there is only one contributor per page and that the standard deviation is about 1.34; most pages has then a single contributor. This point can easily influence page content, increase its obsolescence and reduce its content diversity.

Despite these findings, according to the questionnaire, $85 \%$ of users tend to agree that Stiki can be seen as a training tool for new recruits. For different interviewees, reusing knowledge of experienced employees to form new recruits saves time and ensures staff evolution. One interviewee reported that he often invites his trainees to capitalize their work on Stiki for further reuse.

According to these findings, "Collaborative participation" criterion corresponds to less than "Medium" level in Figure 4. 


\section{Number of contributions and contributors per page}

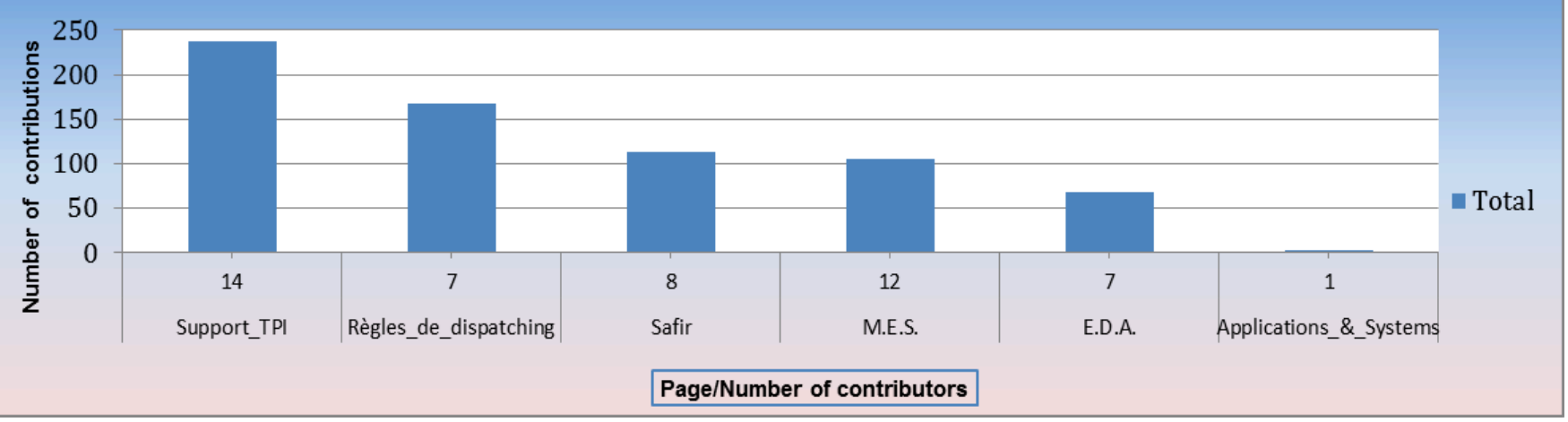

Figure 3 Number of contributions and contributors per page

\section{Q11: Is STMicroelectronics culture in favor of improving knowledge sharing?}

During interviews, some interviewees claimed for including a file converter that converts files formats, for example from a PDF file, to Stiki format. This debate has been launched a few years earlier, but did not lead to any solution. Some people consider that one of the principles of collaborative tools is the willingness to share, so to take time to share their knowledge. Others consider it as a waste of time and seek the fastest way even if it is not compatible with the principle of collaboration. It is a proof of the limitation of open culture in industries where technology reigns and the social aspect is lacking focus. That explains why in Figure 4, "Open culture" level corresponds to "Medium".

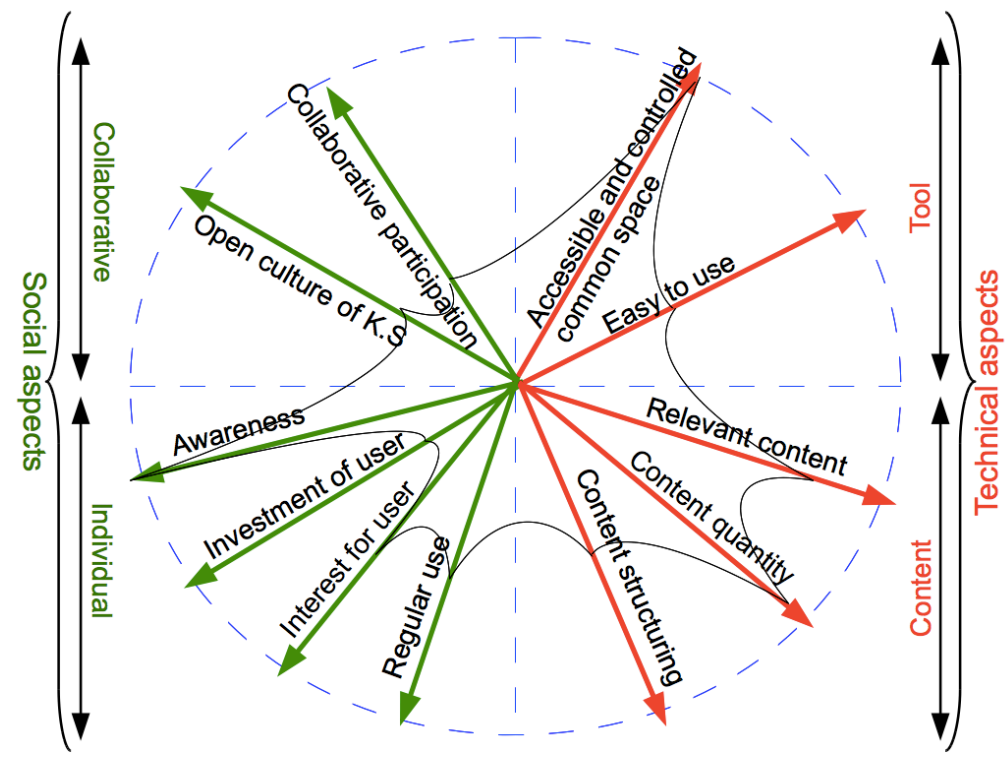

Figure 4 Compliance of Stiki with the criteria that it must meet for knowledge sharing

Is Stiki accomplishing its objectives? Figure 4 summarizes the compliance of Stiki with different criteria, defined in Figure 1. With such presentation, the areas of progress are clearly identified. Therefore, in order to provide a complete Wiki that can meet defined criteria and can accomplish its objectives for knowledge sharing, some progress areas are proposed in the following section. Before that, we discuss the adopted evaluation approach.

\section{Evaluation discussion and limitations}

Despite the care we took with proposed evaluation method, some limits have been identified. For example, the inability to get detailed information that could enrich assessment results. Actually, in Stiki, data about pages creation as well as users' manipulation are not really sufficient to extract important advanced information from the database and better analyze its use. For instance, it wasn't possible to determine the average of consultation duration per page. This measure could be used to identify pages with single reader and their interest for users, in addition to the measure indicating the number of contributors per page. 
Moreover, realizing such an assessment experiment, particularly interviews, is not a usual procedure within STMicroelectronics Company. To ensure credibility and objectivity of results, characteristics such as context and culture are considered and interviews as well as extracted information from the database remained anonymous.

\section{Proposed areas of progress}

Thanks to proposed assessment methods, particularly interviews and questionnaire, some areas of progress proposed by users were identified. In addition, based on some Wiki' experiences relied on literature; we were able to position proposals. In the following, areas of progress according to groups of identified criteria are detailed:

\section{Tool criteria}

During assessment process and particularly during interviews, 5 interviewees have not been trained on use of Stiki, mainly due to their absence at implementation launch. Despite this, there was no other training or information for those who were absent. Nevertheless, the majority is regularly confronted with the use of Stiki. Some people make effort to learn alone; some others use it in their own way. Then, regular training can be an incentive to learn using Stiki and can consequently resolve technical issues with its use. For example, Stiki offers to its users a subscription system to particular domain so to notify them when a page is modified or created. This functionality reduces search time and let people be up-to-date with recent changes. However, most users do not know this feature.

In addition, current version of Stiki dates for 5 years ago. Since this version, several features have been integrated in MediaWiki package. We believe that most of them are useful for Stiki users to find interest in its use. Updating the current version and adding new functionalities are required. Unfortunately, as Stiki does not have an administrator managing its use, it seems less obvious to do it, especially when creating knowledge sharing culture is still a challenge. That is why it is important to sensitize people about the importance of knowledge sharing in organizations.

\section{Content criteria}

Content quantity and content structuring are generally less studied despite their importance. For example, content structuring could provide a common formalism to standardize pages content. Systematizing the evaluation of Stiki is a way to maintain its effectiveness. For instance, set up some indicators about the relevance of publications in terms of content and structure. For example, the number of readers per page, time spent to visit and read one page, assigned scores or comments posted on pages by their readers, etc.

Besides, to avoid the obsolescence of existing pages, it is interesting to tag pages that need to be updated. This requires participation of all users. Then when a user perceives obsolete information, he has to take the initiative to update it if he is the appropriate person to do it or to contact the appropriate person. It seems to be an obvious reaction, but, during evaluation process, we discovered that users' culture in such an organization lacks of social aspects and good practices. For example, when interviewees are asked about their reaction when they find missed information in Stiki, most of them confirmed that they do not correct it directly, but prefer contact the author, even if they have the right answer.

\section{Individual criteria}

Regular use was not studied by any application case in literature because of the difficulty to have results based on a long time period. Regular use can help users to better manipulate the Wiki, ensure its relevance content and eventually avoid its obsolescence. Regularity of use concerns consultation as well as contribution. Like any collaborative tool, using Stiki is mainly based on the goodwill of its users. In the experiment of (Ayres \& Garcia-Perez, 2010), it is clear that, in the short term, proposing competition and a gift to the best contributor may work, but in the long term, this is an unconvincing way. This proves that there are other problems more related to awareness, motivation and willingness of users of such collaborative tool. Improving such criteria is more related to practical activities. In fact, willingness to share is reflected in practice (Ayres \& Garcia-Perez, 2010). Learning to use Stiki collaboratively improves relationship between colleagues and consequently provides a friendly work environment. In that way, each one could feel involved and become aware of the importance of such environment based on team collaboration to share knowledge.

\section{Collaborative criteria}

Firstly, a way that can motivate users is to demonstrate the effectiveness of such a tool in accelerating work and reducing time spent in searching for information. This can be achieved by expanding its application not only in the ST network but also with external collaborators, while managing shareable knowledge. Exchanging knowledge between different teams from different sectors or sites makes easier the problems' understanding (Karolien, 2008; Lykourentzou, et al., 2010). Secondly, as suggested by some interviewees, integrating the documentation of Stiki in projects life cycle like any other mission will promote its use. One interviewee began to apply this idea, but he has encountered some difficulties in updating information during the project progress. Indeed, he writes and shares his contribution throughout the project, but without knowing that Stiki offers a "draft" option to finish his contributions and decide the time of its publication. Apart from this, he believes that integrating documentation of Stiki in the project life 
cycle is easier and more effective in terms of time spent and relevance of information. Such a work methodology seems to be obvious, as well, but we noted during the evaluation process that it exists without being applied. This is due to the lack of awareness and investment of users, as well as some limits in projects supervision. This solution should be investigated in future. Integrating such work methodologies can be limited by the inability of organizations to change. A methodology will be developed following human factors principles in a way that enables them to quickly embrace the novelty and effectively act on it.

\section{Conclusion}

A user-centered approach was developed to evaluate a Wiki for knowledge sharing at STMicroelectronics. It has allowed verifying that Stiki is a useful tool for knowledge sharing even if it can be improved from the social point of view (collaboration, open culture, users' investment, etc.). Stiki is increasingly used as the reference tool, we can deduce its success to ensure an effective knowledge sharing and collaborative work, to take awareness of the importance of such a task and to provide an evolutionary knowledge base maintained by its users. However, based on the evaluation obtained results, some areas of progress have been identified in order to improve its use, to support users' working and to provide an efficient environment for knowledge sharing. For example, structuring content will guide users while sharing knowledge, and expanding its scope of application will enrich its content and improve the collaborative work within the organization.

Despite the care we took with our proposed evaluation method, some limits have been identified as discussed in the evaluation discussion section. For example, the inability to get detailed information that could enrich the assessment results. Besides, we reflected on the possible validity threats to the generalizability of our approach to other settings. Clearly, we designed a proposal for an evaluation method in the context of STMicroelectronics and one might think it's too specific to STMicroelectronics and might not be applicable to other companies and organizational contexts. (Seddon \& Scheepers, 2011) suggests it might be possible to expect that the approach might work in companies that are different but share some contextual characteristics with STMicroelectronics. Actually, STMicroelectronics is a "standard" company that is similar to many organizations sharing the same strategies, culture or structures. Our experimental evaluation approach is complete, since it includes qualitative and quantitative methods ensuring representative results and making it well applicable to other global companies that experience problems similar to STMicroelectronics and that look for similar solutions. We consider it future work to evaluate the applicability of our user centered approach in further case studies within STMicroelectronics and also in other contexts.

On the one hand, involving practitioners throughout our evaluation approach supports their professional values and roles. Working together and sharing knowledge in an appropriate way, through the use of a Wiki for example, is also crucial to joint working. Practitioners need to recognize how others can benefit from such a process. On the other hand, it will be particularly relevant for researchers to focus on studies and approaches of joint working, to develop ways of identifying and qualitatively and quantitatively evaluating its outcomes for users and to understand organizational phenomena. Actually, applying such an approach, involving users in systems' use evaluation, enables researchers and practitioners to understand and address the problems inherent in developing and successfully implementing systems within organizations and then bridge the gap between theory and practice.

Thanks to the richness of adopted evaluation methods and the results obtained, the short-term goal is to take into account the results to improve the use of Wiki for knowledge sharing and hopefully generalizing this to all systems of knowledge sharing. This may be by introducing (Lamb, 2004) quality indicators or an assessment method, containing a regular questionnaire for example. One should note that methods of the proposed evaluation process are reusable to assess regularly not only a Wiki for knowledge sharing but also other tools. In addition, we aim at improving the proposed page structure described in section 3 in order to adapt it to various uses and encourage users to adopt Stiki for knowledge sharing. In the long-term, our goal is to integrate Stiki to a knowledge management project in an existing information system where it can serve as a tool for knowledge capitalization and sharing. To this end, the first stage will consist of its integration in project management where we aim at implementing a process of continuous improvement of safety and ergonomics based on collaborative knowledge gathering, issues identification and solution development. 


\section{Bibliography}

Avram, G., 2006. At the Crossroads of Knowledge Management and Social Software. The Electronic Journal of Knowledge Management, 4(1), pp. 1-10.

Ayres, R. \& Garcia-Perez, A., 2010. Wikifailure : the Limitations of Technology for Knowledge Sharing. Electronic Journal of Knowledge Management, 8(1), pp. 43-52.

Brooke, J., 1996. SUS - A quick and dirty usability scale, s.I.: Usability Evaluation in Industry.

Conte, A., Hassine, I., Giraudin, J.-P. \& Rieu, D., 2001. AGAP : un Atelier de Gestion et d'Application de Patrons. s.I., Actes du XIXème Congrès INFORSID.

Duyck, J.-Y., 2003. Des lettres et des chiffres : vers la "troisième génération" du qualitatif en. Revue Science de Gestion, Volume 30, pp. 179-206.

Fonteyn, M. \& Bauer-Wu, S., 2005. Using qualitative evaluation in a feasibility study to improve and refine a complementary therapy intervention prior to subsequent research. Complementary Therapies in Clinical Practice, pp. $247-252$.

Gee-Woo, B., Robert, Z., Young-G, K. \& Jae-Nam, L., 2005. Behavioral Intention Formation in Knowledge Sharing: Examining the Roles of Extrinsic Motivators, Social-psychological Factors, and Organizational Climate. MIS Quarterly, 29(1), pp. 87-111.

Kaplan-Leiserson, E., 2004. We Learning: Social Software and E-Learning-Part2. [Online] Available at: http://www.learningcircuits.org/2004/jan2004/kaplan2.htm

Karolien, S., 2008. Putting Library 2 . 0 into practice : the process of setting up a wiki as a tool for knowledge sharing in a public library. [Online]

Available at: Public Library of Vlissingen

Krebs, M., Ludwig, M. \& Müller, W., 2010. Learning Mathematics using a Wiki. Procedia Social and Behavioral Sciences, Volume 2, p. 1469-1476.

Lamb, B., 2004. Wide open spaces: Wikis, ready or not.. EDUCAUSE Review, 39(5), pp. 36-48.

Lykourentzou, I. et al., 2010. CorpWiki: A self-regulating wiki to promote corporate collective intelligence through expert peer matching. Information Sciences, pp. 18-38.

MacManus, R., 2006. eBay Wiki-world's largest commercial wiki launched. [Online] Available at: www.readwriteweb.com

Montero-Fleta, B. \& Perez-Sabater, C., 2011. Knowledge construction and knowledge sharing: a Wiki-based approach. Procedia - Social and Behavioral Sciences, pp. 622-627.

Parker Kevin \& Chao, J., 2007. Wiki as a Teaching Tool. Interdisciplinary Journal of Knowledge and Learning Objects, Volume 3, pp. 1-16.

Raitman, R., Augar, N. \& Zhou, W., 2005. Employing Wikis for Online Collaboration in the E-Learning Environment: Case Study. hird International Conference on Information Technology and Applications, pp. 142-146.

Ramanau, R. \& Fawei, G., 2009. Researching the use of Wiki's to facilitate group work. Social and Behavioral Sciences, pp. 2620-2626.

Sandelowski, M., 1996. Focus on qualitative methods: using qualitative methods in intervention studies. Res Nurs Health, pp. 359-364.

Seddon, P. \& Scheepers, R., 2011. Towards the improved treatment of generalization of knowledge claims in is research: drawing general conclusions from samples. EJIS, pp. 1-16.

Tay Pei Lyn, G., 2009. Wikis as a knowledge management tool. Journal of Knowledge Management, 13(4), pp. 64-74. Teck Heng, L. \& Marimuthu, R., 2011. Let's Wiki in Class. Procedia - Social and Behavioral Sciences, Volume 67, pp. 269-274. 